\title{
3D Reconstruction of Protein Kinase C Molecules From Electron Micrographs Of Two-Dimensional Crystals On Lipid Monolayers In Plane Group p1
}

\author{
A.S. Solodukhin*, R.H. Kretsinger**, and J.J. Sando* \\ *The Department of Anesthesiology, University of Virginia Health System, P.O. Box 800710, \\ Charlottesville, VA, 22908 \\ **The Department of Biology, University of Virginia, P.O. Box 400328, Charlottesville, VA, 22904
}

Analysis of tilted images of two-dimensional (2D) protein crystals by electron microscopy is a well established procedure for determination of three-dimensional (3D) structure [1]. Usually 2D crystals of proteins display p2, p3, p4 or p6 plane group symmetry. These symmetries define an origin and place constraints on the relative phase angles of the reflections computed by Fourier transform of the observed images. An exception is the case of space group p1, for which any image and any origin could be used to begin with (since there is no symmetry). We discuss some difficulties in 3D structure determination that arise in plane group 1, using the example of protein kinase $\mathrm{C}$ (PKC) and its regulatory domain $(\mathrm{RD})$.

Protein kinases C (PKC) are a family of eleven signal-transducing isozymes that are activated by their interactions with membrane lipid components following stimulation of transmembrane receptors by hormones, neurotransmitters, and growth factors. Since different isozymes are implicated in different signal transduction pathways or diseases, drug design must be targeted to individual isozymes. Progress in development of isozyme-selective drugs has been slow in part because a 3D structure of intact PKC has yet to be determined. A few 3D crystal structures of portions of PKC - the $\mathrm{C} 2$ domain of PKC and, more recently, the catalytic domain of PKC - have been reported [2-3]. However, all attempts to grow 3D crystals of intact PKC have failed.

2D crystals of PKC and RD were grown on lipid monolayers composed of dioleoylphosphatidylcholine: dioleoylphosphatidylserine: diolein (45:50:5, molar ratio). Images were taken with a TECNAI-12 electron microscope at acceleration voltage of $100 \mathrm{keV}$, magnification of $67000^{\mathrm{x}}$, and at tilt angles of +55 to -50 . Analysis of projection maps of untilted images revealed that the unit cells of both PKC and RD have sides a $\mathrm{b}$ and inter-edge angle = $120^{\circ}$. RD has an edge length of $331 \AA$, whereas intact PKC has an edge length of $46 \quad 1 \AA$ [4]. The unit cell parameters suggest P3 symmetry. Calculations with programs (e.g. CRISP) designed to discriminate between P1 and higher order plane groups provided phase residuals for P3 symmetry less than $25^{\circ}$ and showed a clear minimum in comparison with other groups [5]. However, the unit cell area is not enough to accommodate more than one molecule and nothing about the sequence suggests p3 symmetry.

Additional analysis by calculation of Fourier Rings Correlation (FRC) and Difference Phase Residuals (DPR) performed for all untilted projection maps shows that, although $(0,1),(1,0)$ and $(1,-1)$ reflections show similar, but not identical amplitudes, it is possible to distinguish single orientation of one crystal relative to another one when FRC is maximal and DFR is minimal (Fig.1). That means that data must be processed in 1 symmetry and only crystals with the same orientation must be merged in reciprocal space. For p1 a phase origin is arbitrary, so in order to set a phase origin for all untilted images in the same manner, the following technique has been used: p3 
symmetry is applied for initial projection, then calculated $\mathrm{H}$ and $\mathrm{K}$ optimal shifts are manually set for the p1 symmetry image.

FRC and DPR analysis also showed that some untilted projections have low FRC and high DPR; thus, only projections with good FRC and DFR were merged to create untilted, core data sets. Data from tilted films were then added using low tilt angles first, high tilt angles last. Crystals rotated by $180^{\circ}$, in the plane of the 2D lattice, with respect to the core data set were identified by phase residual comparisons after merging rotated or unrotated data with the core data set in reciprocal space. The problem of determining the correct phase origin in three-dimensions will be discussed. Because the regulatory domain confers membrane attachment, strong inferences can be made about the orientation of the intact PKC molecule relative to the lipid monolayer.

\section{References}

[1] L.A. Amos et al., Prog Biophys Molec Biol 39 (1982) 183

[2] G. Zang et al., Cell 81 (1995) 917.

[3] Zhang-Bao Xu et al., J Biol Chem 279 (2004) 50401.

[4] A.S. Solodukhin et al., Biophys J 82 (2002) 2700.

[5] S. Hovmöller, Ultramicroscopy 41 (1992) 121.

[6] This research was supported by HHS grant GM31184.


Fig.1. Fourier Rings Correlation (FRC) and Difference Phase Residuals (DPR) of 3 possible orientations of 2D crystal A relative to the crystal B. a) good correlation, orientation angle 0 ; b) and c) orientation of one crystal changed by 60 and 120 respectively. 